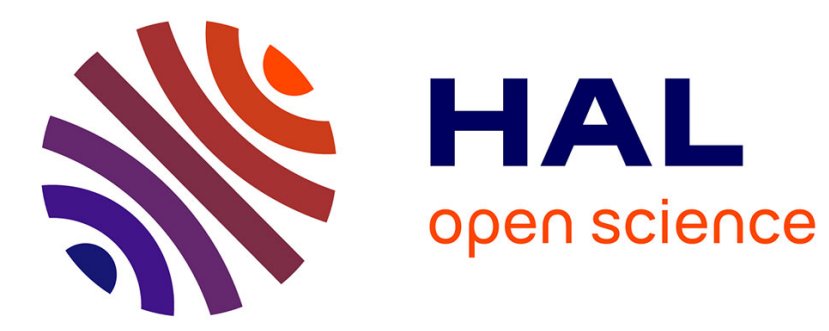

\title{
Experimental study of brittle fracture of heterogeneous model systems
}

\author{
D. Lely, S. Roux
}

\section{To cite this version:}

D. Lely, S. Roux. Experimental study of brittle fracture of heterogeneous model systems. Journal de Physique III, 1991, 1 (10), pp.1675-1684. 10.1051/jp3:1991221 . jpa-00248691

\section{HAL Id: jpa-00248691 https://hal.science/jpa-00248691}

Submitted on 1 Jan 1991

HAL is a multi-disciplinary open access archive for the deposit and dissemination of scientific research documents, whether they are published or not. The documents may come from teaching and research institutions in France or abroad, or from public or private research centers.
L'archive ouverte pluridisciplinaire HAL, est destinée au dépôt et à la diffusion de documents scientifiques de niveau recherche, publiés ou non, émanant des établissements d'enseignement et de recherche français ou étrangers, des laboratoires publics ou privés. 
Classification

Physics Abstracts

$62.20 \mathrm{M}-81.40 \mathrm{~N}$

\title{
Experimental study of brittle fracture of heterogeneous model systems
}

\author{
D. Lely and S. Roux (*)
}

Service de Mécanique des Matériaux et des Structures, IMA, Faculté Polytechnique de Mons, Rue de Joncquois, 7000 Mons, Belgium

Centre d'Enseignement et de Recherche en Analyse des Matériaux, Ecole Nationale des Ponts et Chaussées, Central IV, 1 Av. Montaigne, F-93167 Noisy-le-Grand Cedex, France

(Received 19 April 1991, revised 31 May 1991, accepted 14 June 199I)

\begin{abstract}
Résumé. - Nous présentons des résultats expérimentaux concernant la fracture fragile de milieux modèles: plaques de plexiglass percées de trous de tailles aléatoires, où le désordre est bien contrôlé. Nous nous intéressons plus particulièrement à la morphologie de la fissure. Nous trouvons un accord remarquable entre la conformation de la fissure et celle du chemin minimal qui traverse le milieu. Ce résultat conforte une analyse antérieure de Jeulin [1] et suggère un caractère auto-affine des fissures dans un milieu fragile hétérogène comme conjecturé récemment à partir de simulations numériques [2].
\end{abstract}

\begin{abstract}
We report on some experimental results obtained for the fracture of model systems, plexiglass plates, with a random distribution of holes of various sizes, where the disorder is well controlled. We focused our attention on the geometrical properties of the fracture. In particular, we find a remarkable agreement, between the main crack and the minimal path which crosses the plates. This result supports some previous analysis of such correlations by Jeulin [1] and suggests a self-affine character of the cracks in heterogeneous brittle materials as recently suggested from numerical simulations [2].
\end{abstract}

\section{Introduction.}

Some recent studies of fracture of disordered brittle materials using the tools of statistical mechanics have highlighted the determining role of disorder in this phenomenon [3]. This problem is not new. However, recent progress has been achieved recently in particular from numerical simulations. Most analytical approaches in this field usually focus on the behavior of the first defect to fail in the material, assuming that this first event is enough to trigger an unstable crack propagation. In the classical work of Weibull [4] for instance the weakest defect in the material was considered - together with some additional hypothesis which can be generalized. Other approaches such as that of Duxbury et al. [5] favor the role of the stress

(*) Also at Laboratoire de Physique et Mécanique des Milieux Hétérogènes, URA CNRS 857, Ecole Supérieure de Physique et Chimie Industrielles, 10 rue Vauquelin, F-75231 Paris Cedex 05, France. 
distributions, by considering the element which carries the largest load. Although these two approaches give some insight in the initial stage of fracture, depending on the disorder (e.g. defect size distribution) within the material, it may happen that the breaking strength of the material is reached after a large number of failures of individual elements [6]. In such a case, a theoretical description of the damage process should take into account the interaction of microcracks, and thus it should also make use of a satisfactory description of the spatial distribution of these microcracks. With our present knowledge, it has not been possible to meet this challenging requirement through analytical tools for a realistic geometry. Thus one has to use numerical simulations or model experiments.

This limitation becomes even more drastic when one is interested not only in the mechanical properties of the system (such as the strength) but also in the geometrical features of the crack distribution, or of the main crack. In this case, whatever is the disorder, interactions between defects are relevant and cannot be neglected. In this paper we report on an experimental approach to this problem. In most circumstances, the fracture path of an heterogeneous material is not a straight plane but rather a meandering surface with some roughness, such that the surface makes use of the defects present within the material. The statistical description of this roughness is the major aim of this study.

\section{Experimental approach.}

In order to connect some fracture properties to the local geometry, it is important to have a simple and well controlled system. We thus consider plexiglass plates which are brittle in the conditions of the experiment. The size of the plates is $210 \mathrm{~mm} \times 150 \mathrm{~mm}$ with a thickness of $3 \mathrm{~mm}$. In these plates, we punched circular holes whose centers lie on the sites of a regular square lattice whose principal axes are oriented at $45^{\circ}$ with respect to the borders of the plates. The distance between sites in this lattice is $10 \mathrm{~mm}$. Although the centers of the holes are regularly distributed, their diameters are randomly sampled among three sizes 5,7 and $9 \mathrm{~mm}$, with probabilities $p_{1}, p_{2}$ and $p_{3}$ respectively. A typical example is shown in figure 1 . The number of holes in one plate is 221 .

Extreme care has been taken in drilling the holes in the plates (with a digital drilling machine) so as to avoid small defects on the border which may initiate cracks in the medium.

A total of 32 plates have been studied. The samples have been shared into 4 series called A, B, C and D, each being characterized by a different set of probabilities as indicated in table $\mathbf{I}$, which also indicates the number of samples in each series.

The plates were subjected to a simple traction in a testing machine, where a slow displacement rate is imposed $(0.1 \mathrm{~mm} / \mathrm{min}$ ) (see Fig. 2). The plates were attached through two grips which were fixed to the testing machine in such a way that either a free rotation could take place, or rotation was impelled. In the first case, the force-displacement relation could be recorded safely, but once half of the plate was broken the rotation of the grips was rather large and the end of the crack systematically deviated from the initial trajectory. After having blocked the rotation of the grips, this spurious effect disappeared, but the friction of the grip on the guide was large and the measured force-displacement relations were no longer reliable. Some preliminary tests were performed with the free rotation, but all samples referenced in table I were fractured with no rotation.

Let us comment the few measured force displacement characteristics, in the preliminary tests. An example is shown in figure 3. The curve consists of a few peaks which can easily be identified as failures of individual ligaments between holes. The peak stress was sometimes found for the first bond broken, and sometimes for a larger number (see e.g. Fig. 3 where the maximum is reached for the third ligament to break). As the fracture proceeds, these individual failures gather into bursts, or series of ligaments which can no longer be 


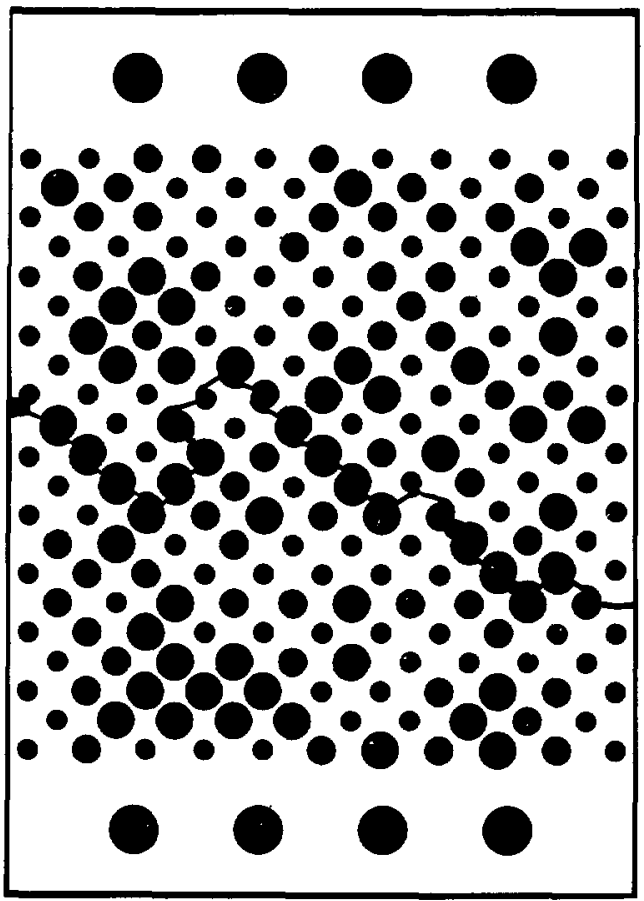

Fig. 1. - Photograph of one of our sample plates. The four holes at the top and the bottom of the plate are used to hold the plate during the experiment. In the plate a series of holes has been drilled, with a random distribution of radii. The plate is fractured along the black curve connecting holes. We argue that, on average, a good statistical approximation to the location of the crack is given by the path which minimizes the intersection with the material.

Table I. - The table indicates for the four series of plates A, B, C and D, the probabilities $p_{1}, p_{2}$ and $p_{3}$ of having a hole of size 5,7 and $9 \mathrm{~mm}$, respectively, and the number of plates in each series.

\begin{tabular}{|c|c|c|c|c|}
\hline Series & Samples & $p_{1}$ & $p_{2}$ & $p_{3}$ \\
\hline A & 10 & $1 / 2$ & $1 / 4$ & $1 / 4$ \\
\hline B & 8 & $7 / 15$ & $1 / 3$ & $1 / 5$ \\
\hline C & 7 & $3 / 8$ & $3 / 8$ & $1 / 4$ \\
\hline D & 7 & $5 / 12$ & $1 / 4$ & $1 / 3$ \\
\hline
\end{tabular}

distinguished individually on the characteristics. All along the characteristic, the behavior was elastic linear between the peaks, and no plastic deformation could be observed. Due to the large statistical fluctuations occurring between different plates having the same set of probabilities (same probability distribution, but not identical holes), and the few number of characteristics generated, it has not been possible to extract from the experiment any 


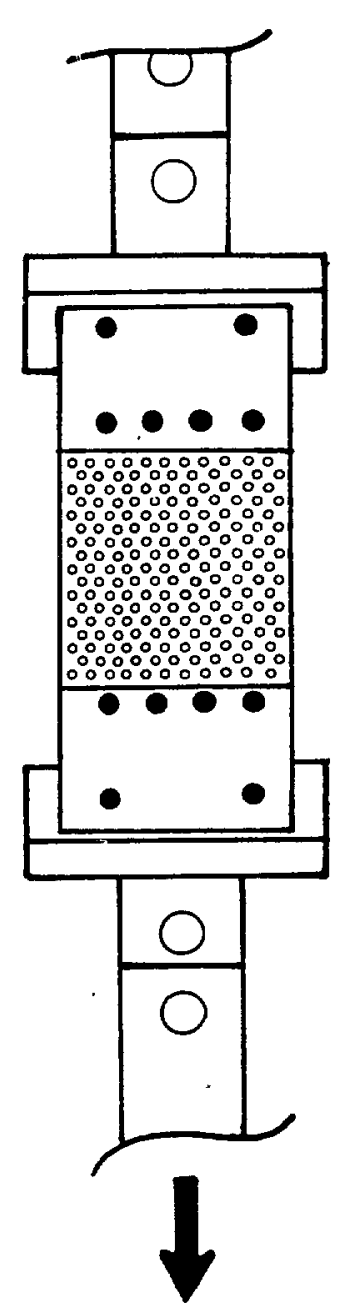

Fig. 2. - Schematic plot of the sample in the testing machine.

meaningful statistical information. We thus concentrated on the purely geometrical study of the main crack geometry.

The spirit of our approach is to try to connect the main crack to the " minimal path " (i.e. the path which minimizes the length of the intersection with the solid phase). A similar approach was followed by Jeulin [1] experimentally and by Hansen et al. [2] through numerical simulations. Let us first recall a few properties of the minimal problem which comes from a very different framework.

\section{Minimal path problem.}

Finding the minimal path in a random medium is a very classical problem of statistical mechanics. The basic problem has been studied first for the boundaries of spin domains in random-exchange Ising models [7]. It has been recently established that this problem could be mapped onto very different ones, including models of stochastic growth, deposition of particles, conformation of directed polymer in a random medium at zero temperature, 


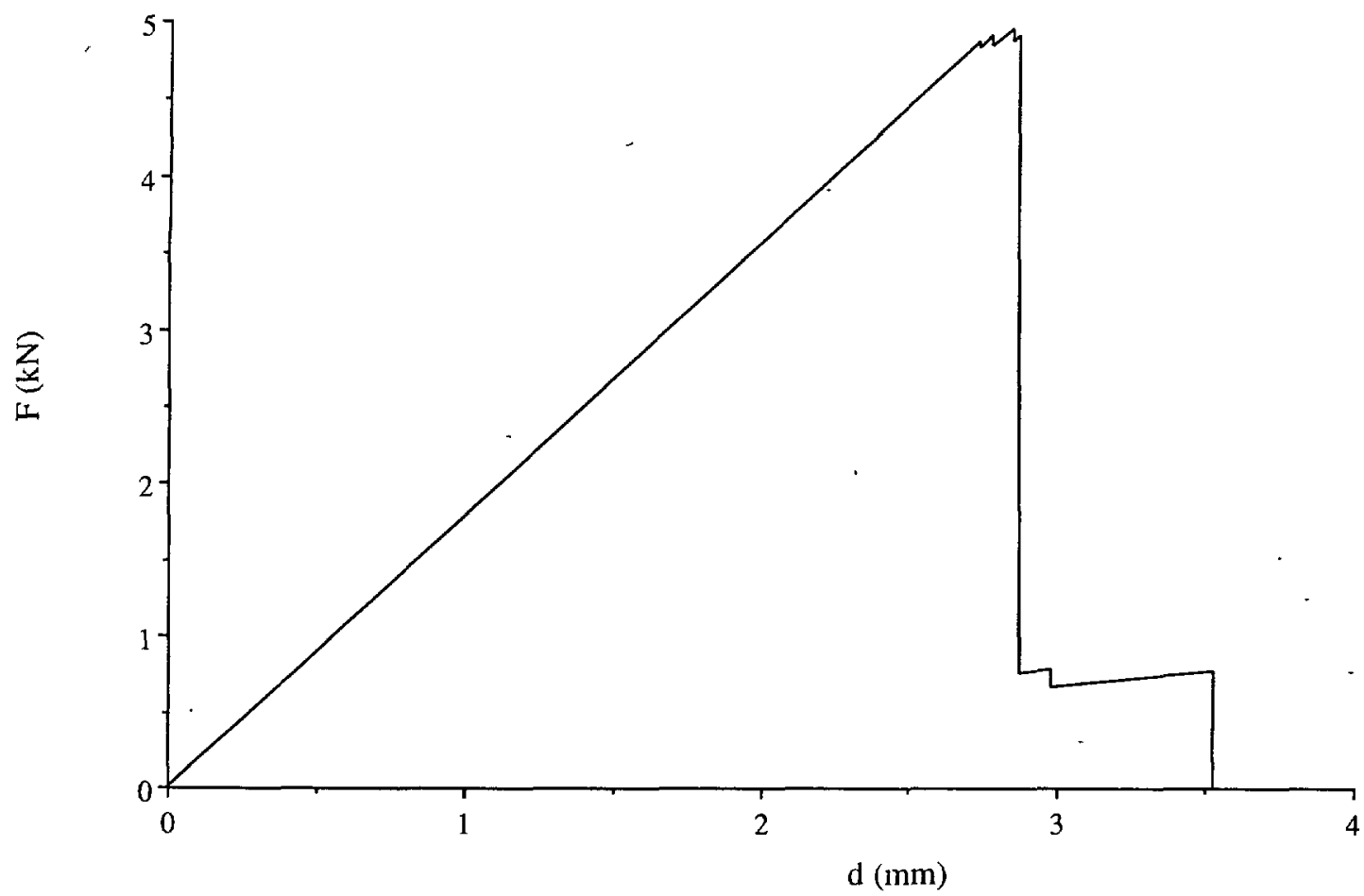

Fig. 3. - Typical force-displacement relation obtained experimentally. Note that the peak stress is not reached for the breaking of the first bond, but only for the third bond to break.

Burgers' equation with noise, ... These recent works allowed to obtain some exact scaling properties of the minimal path problem in two dimensions [8], and a lot of numerical results concerning the three-dimensional case [9].

Let us consider a lattice whose bonds $i$ are attributed a random weight $a_{i}$ independently chosen from a statistical distribution $p(a)$. These weights are uncorrelated. No restriction is assumed on the distribution of weights provided they are positive [10]. We consider all the paths $P$ which cross the lattice from one border parallel to the $y$-axis to the opposite one. A path has no branching. In general it may contain some backward steps (overhangs), however, no large-scale overhangs can occur, and thus restricting the paths to be directed should not affect the scaling properties reported below. The mean direction of the path is thus parallel to the $x$ axis. A basic quantity of interest is the "energy " $E$, or "effective lengh » of the path. It is defined as the sum over all sites visited by the path $P$ of the local weights $a_{i}$

$$
E(P)=\sum_{i \in P} a_{i}
$$

The minimal path is the one which minimizes this energy. The main contribution to the energy is a term which is proportional to the length of the path, $L$, so that the energy per unit length of the path tends to a constant when the length goes to infinity. However, the first correction to this trend is non-analytic in $L$. It can be written

$$
\langle E(L)\rangle=e L+A L^{x}+\text { h.o.t. }
$$

where $x=1 / 3$ in two dimensions, and $A$ is a lattice-dependent constant. The fluctuation of $E(L)$ around its mean, $\sigma(L)=\left(\left\langle E^{2}(L)\right\rangle-\langle E(L)\rangle^{2}\right)^{1 / 2}$, also scales as $\sigma(L) \propto L^{x}$. 
Another set of properties concerns the geometrical features of the path. If we impose that the path is on average oriented along the $x$ direction (e.g. if it connects two opposite borders perpendicular to $x$ in a lattice), then one can define the roughness of the path $w$, as the root mean square deviation of the path from its average direction. If $y$ is an axis perpendicular to $x$, and $y(x)$ gives the equation of the path, the roughness $w$ is defined through

$$
w^{2}=(1 / L) \int y^{2}(x) \mathrm{d} x-\left((1 / L) \int y(x) \mathrm{d} x\right)^{2}
$$

where $L$ is the length of the path.

If the path were simply a random path then the roughness would vary as the square root of its length. For the minimal path, the roughness is much larger. It scales with the length of the path as

$$
w \sim L^{\zeta}
$$

where in two dimensions, $\zeta=2 / 3$ [8]. This type of scaling property is refered to as selfaffinity. Let us also note that since $\zeta<1$, the path is asymptotically flat: the relative roughness $w / L$ tends to zero as the system size goes to infinity.

In our problem, the local random variables are simply related to the distance separating two holes in the plate. Thus the local weight of a bond will be the area of material to break in order to create a crack which connects two holes. The " energy " of the path will then be the total area of broken material along the crack.

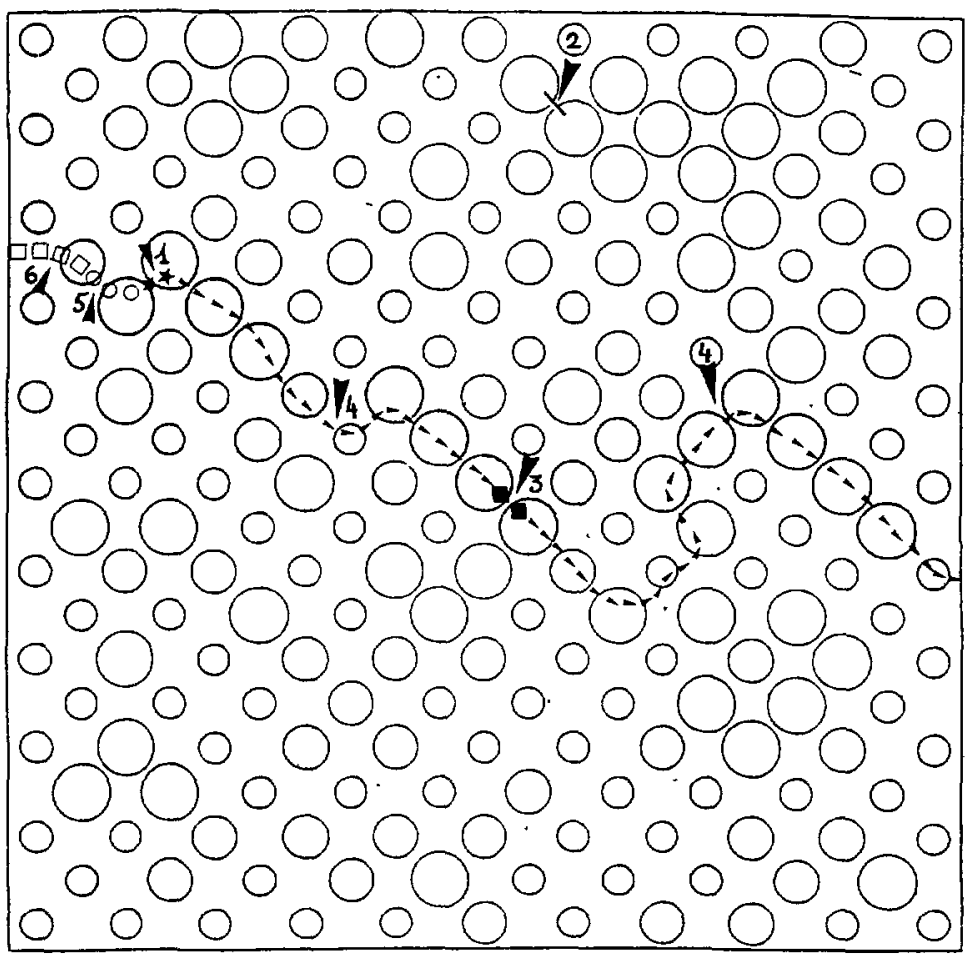

Fig. 4. - Temporal development of the crack for the plate of figure 1. Note the breaking of bonds (2) which are not connected to the rest of the crack. The numbers indicate the order in which the ligaments were broken. Each number corresponds to a peak in the force displacement relation of figure 2. 
This type of mapping has been used recently in the field of ductile fracture [11] of porous materials where one can show easily using this formulation that the fracture stress will decrease with the porosity to the power $2 / 3$ in three dimensions. However, no such analysis has been performed for brittle fracture, although a few studies have investigated the roughness of cracks [12] or even the fractal character of brittle cracks.

\section{Comparison.}

We have performed some numerical simulations in order to compare the minimal path found in our particular geometry and the fracture path. We used a transfer matrix approach analogous to the one used by Derrida and Vannimenus [7]. We restricted ourselves to directed paths, allowing the path to connect holes only through bonds inclined at 0 and $\pm \pi / 4$ with respect to the $x$-axis. This directedness is commonly believed not to affect the scaling properties of the paths, since large-scale overhangs are unlikely. However, the directedness allows to use much more efficient algorithm than the general non-directed case. After one sweep through the lattice, it is possible this way to compute the length of the minimal path, and a second sweep allows to extract the sites through which the path goes. Due to the fact that the radii of the holes could only assume four distinct values, the minimal paths were not unique (it is possible to find more than one path which reaches the minimal length). Figure 5 displays an example of the minimal paths computed on the geometry of the sample of figure 1 . We emphasis that these simulations do not compute any stress distribution, but only refer to the purely geometrical minimal path defined above.

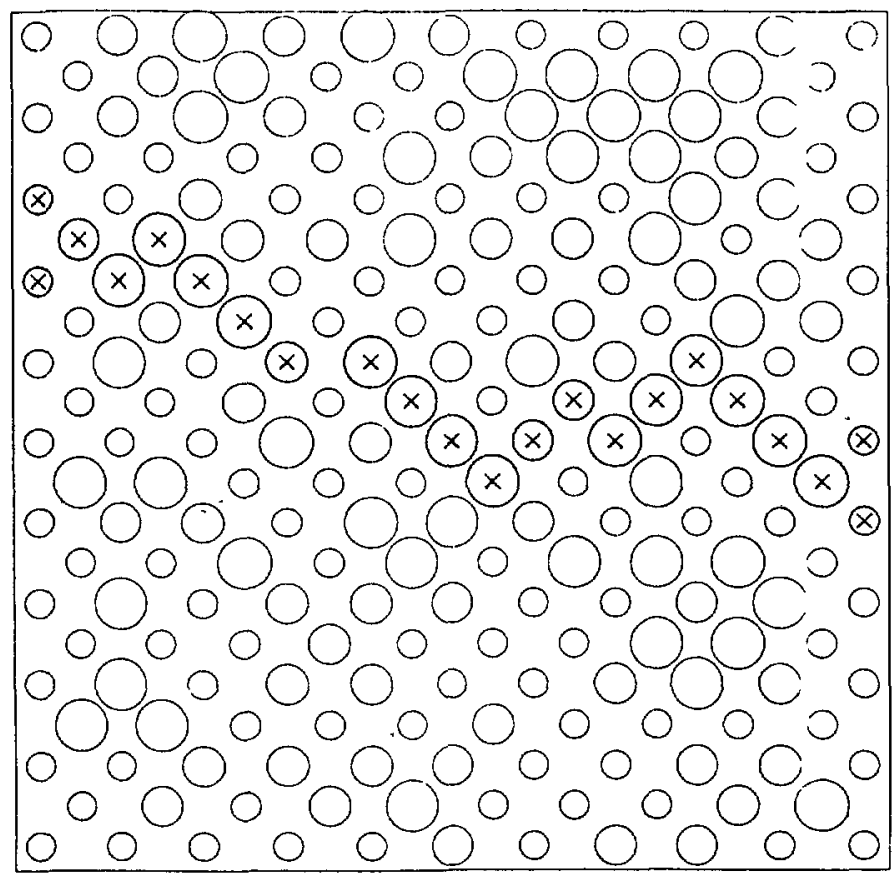

Fig. 5. - Minimal path obtained in the example of the plate from figure 1. A cross in a hole indicates that at least one minimal path goes through this hole. A strong correlation is found with the actual crack pattern (see Fig. 4). 
We have checked in particular by varying the size of the lattice that the expected relations recalled above (mean and standard deviation of the length of the minimal path, and roughness) were verified within numerical uncertainties.

The most crucial test was to compare, for each sample broken, the geometry of the fracture path and that of the minimal path (i.e. we compare the equivalent of Figures 1 and 5 for each sample). To make this comparison quantitative, we computed systematically the length of the paths, and their roughness both for the fracture and the minimal path. Figure 6 shows a scatter plot of both lengths for each sample (all series). A strong correlation is visible from the graph.

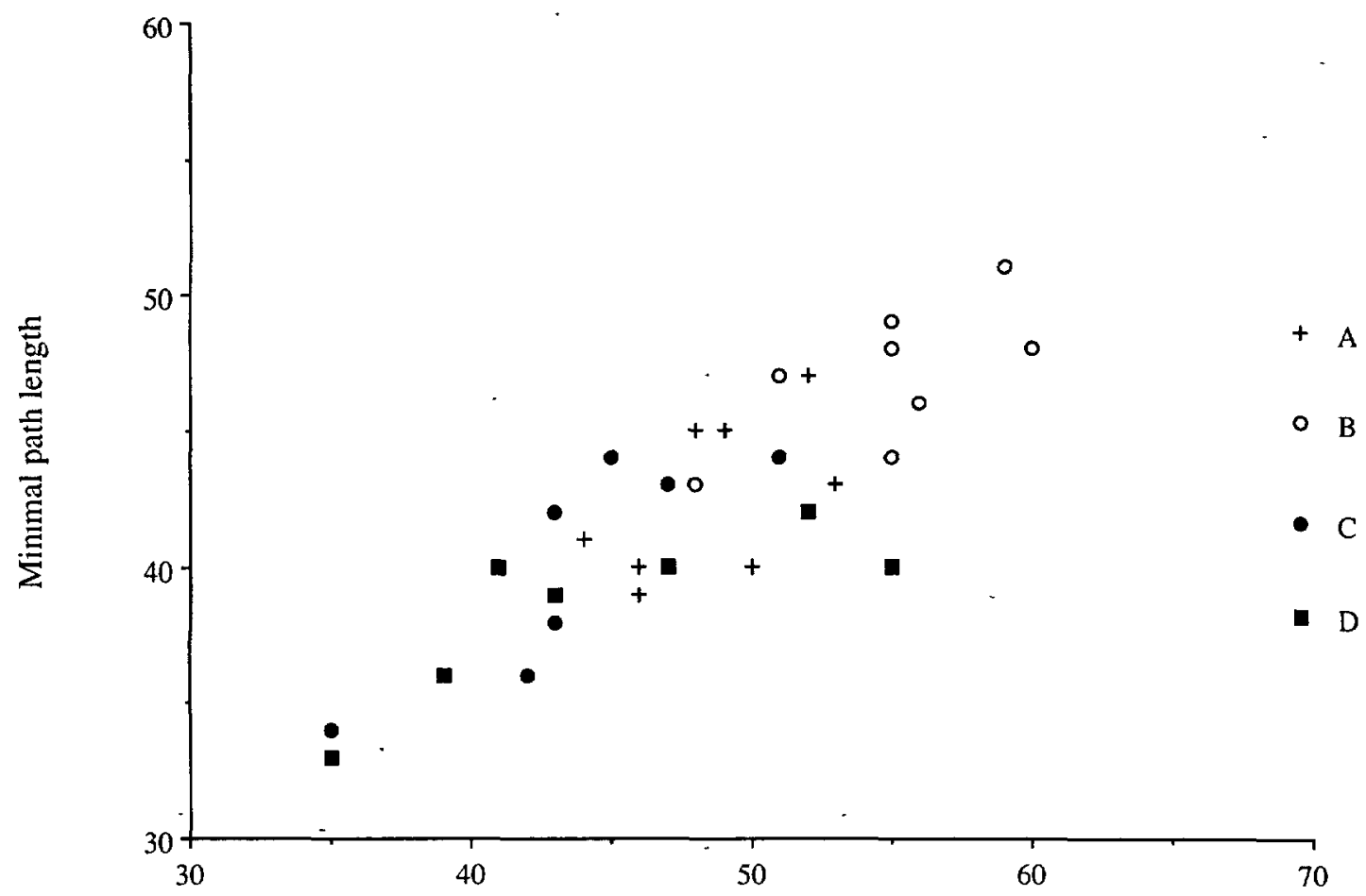

Fracture length

Fig. 6. - Scatter plot of the length of material broken for the minimal path $L_{\mathrm{mp}}$ versus the actual length in the fracture experiments. Each point corresponds to a single sample. The different symbols indicate the series (+) A ; (O) B ; (o) C ; (ש) D.

The data concerning the roughness are more widely scattered. This is due to the fact that a small difference between both paths (a few ligaments which are distincts) can actually produce a large difference in the roughness. In particular as mentioned above, when a free rotation of the grips was possible, then the end of the fracture path markedly and systematically deviates from the minimal path, because of the change in boundary conditions governing the end of the process, which is definitely not taken into account in the minimal path problem. However, when we averaged the roughness over all elements in a given series, then the data reveal a good correlation. This is shown in figure 7.

The length and the roughness are a rather global characterization of the path, in the sense that one could imagine that very different paths can exhibit a similar roughness and almost the 


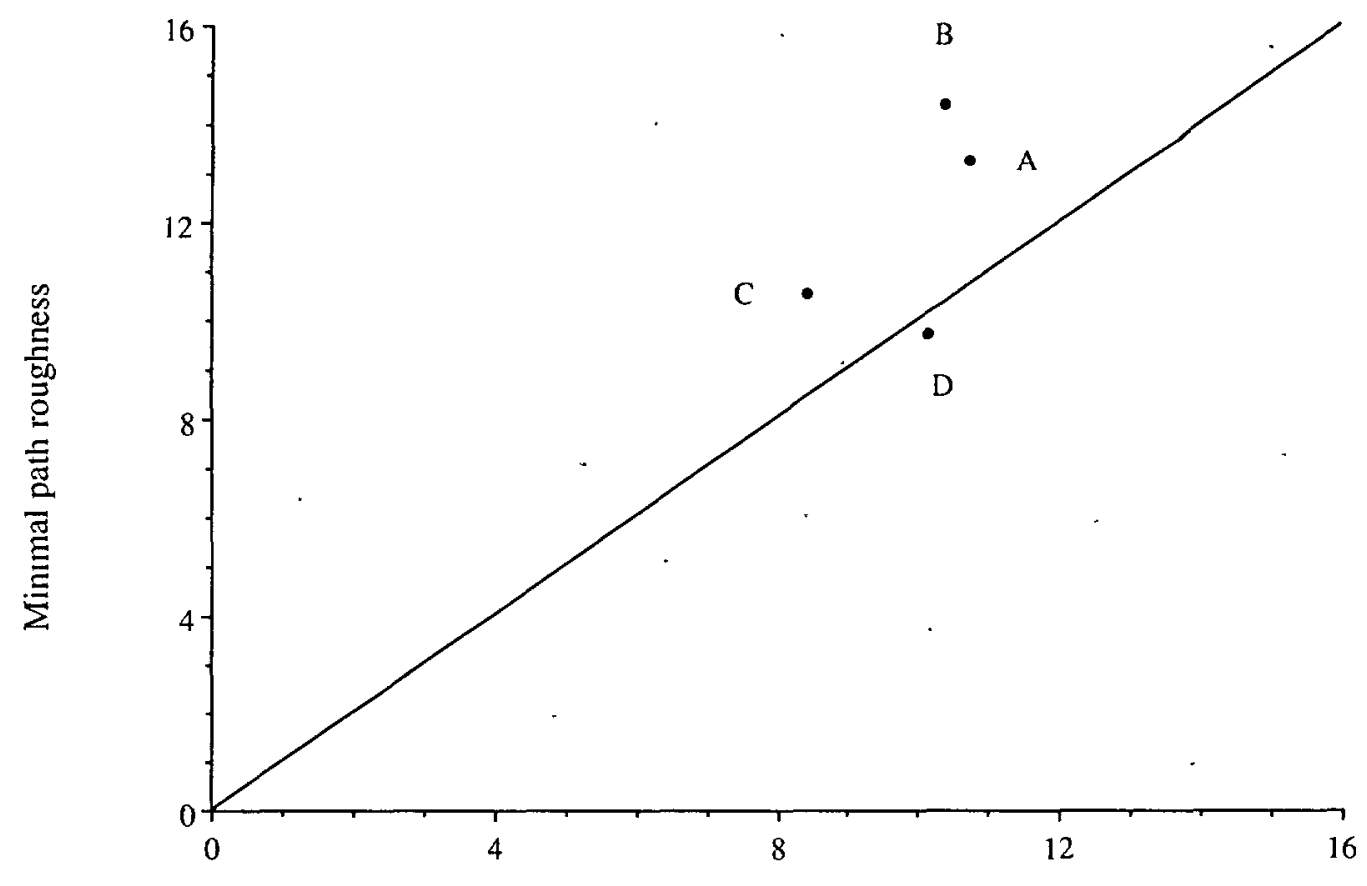

Fracture roughness

Fig. 7. - Plot of the average roughness of the minimal path versus that of the actual fracture. Averages have been taken over all samples in each series. Each point corresponds to a complete series, indicated by a letter nearby each point.

same length. Thus a stronger comparison can be done in computing the proportion of common sites which belong to both paths. The average proportion of common sites is given in table II, for each series.

The first column gives the fraction of common sites shared between the minimal path and the fracture path, averaged over all samples. When averaging over all series, we obtain a fraction of common sites close to $58 \%$. We also note that the minimal path problem is extremely sensitive to noise. If the fracture starts by the breakage of a bond which is remotely

Table II. - The table indicates the fraction of sites which belong to both the fracture and the minimal path for the four series of plates $\mathrm{A}, \mathrm{B}, \mathrm{C}$ and $\mathrm{D}$. The third column (*) is the same percentage computed over the plates where the two paths intersect at least for one site.

\begin{tabular}{|c|c|c|}
\hline Series & Common sites & Common sites $\left(^{*}\right)$ \\
\hline A & $58 \%$ & $65 \%$ \\
\hline B & $40 \%$ & $62 \%$ \\
\hline C & $78 \%$ & $78 \%$ \\
\hline D & $58 \%$ & $58 \%$ \\
\hline
\end{tabular}


located from the minimal path, then the fracture has a fairly low probability to encounter the absolute minimal path, but it may be very close to a short path. Thus if we perform the average of the common sites only over the lattices where a non zero intersection exists, then the percentage increases by a large amount (for series $A$ and $B$ ). The average of overlap between paths including all series with this restriction is about $65 \%$.

In fact, it is obvious that the minimal path can only be considered as an approximation to the true fracture path. However, the fact that the correlation is strong suggests that eventually the geometrical statistical properties concerning the scaling of the roughness can be transposed from one field to the other.

\section{Conclusion.}

We have presented an experimental study of the fracture of a model disordered system. A direct comparison between the fracture path and the minimal path shows a significant overlap which suggest that statistical properties of the geometry of the fracture path may be comparable to that of the minimal path.

The natural continuition of this work is naturally to investigate the scaling behavior of the roughness of fractures on a much larger scale in real materials. However, such a study will certainly not allow a direct one to one comparison with a minimal path, because of the precise knowledge of the microstructure which is needed. That is the reason why it was first necessary to establish a possible connection first on such model systems.

\section{Acknowledgments.}

We thank P. Acker, M. Fremond, D. Jeulin, A. Gilabert and P. Rossi for useful discussions and support. The help of G. Dubuet, Ph. Rossi and J. L. Salhi for the numerical simulations and the experiments is gratefully acknowledged. We acknowledge the support of the ATP "Matériaux Hétérogènes » of the PIRMAT (CNRS).

\section{References}

[1] Jeulin D., Rev. Phys. Appl. 23 (1988) 549.

[2] Hansen A., Hinrichsen E. L. and Roux S., Phys. Rev. Lett. 66 (1991) 2476.

[3] HerrmanN H. J. and Roux S. Eds., Statistical models for the fracture of disordered materials, North-Holland (Amsterdam, 1990) ; J. C. Charmet, S. Roux and E. Guyon, Disorder and fracture, Proceeding of Cargèse, 1989 (Plenum Press, 1990).

[4] Weibull W., J. Appl. Mech. 28 (1951) 293.

[5] Duxbury P.: M., Beale P. D. and Leath P. L., Phys. Rev. Lett. 57 (1986) 1052 ; see also P. M. Duxbury in Ref. [3] (Chapt. 6 and references therein).

[6] De Arcangelis L., Hansen A., Herrmann H. J. and Roux S., Phys. Rev. B 40 (1989) 877 and references therein.

[7] Derrida B. and Vannimenus J., Phys. Rev. B 27 (1983) 4401.

[8] Kardar M., Parisi G. and Zhang Y. C., Phys. Rev. Lett. 56 (1986) 889.

[9] KARDAR M. and ZHANG Y. C., Europhys. Lett. 8 (1989) 233.

[10] Roux S., Hansen A., da Silva L., Lucena L. and Pandey R., to appear in J. Stat. Phys. (Oct. 1991).

[11] Roux S. and François D., Scripta Met. 25 (1991) 1084.

[12] Coster M. and Deschanvres A., 2nd European Symposium on Image Analysis in Material Science, Biology and Medicine, Pract. Met. S 8 (1978) 61 ;

Coster M. and Chermant J. L., "Précis d'analyse d'image " (Presses du CNRS, Paris, 1989) and references therein. 